
\title{
28 Research Soure \\ Vitamin D Deficiency in Children with Acute Bronchiolitis: A Prospective Cross-Sectional Case- Control Study
}

Inbal Golan-Tripto ( $\square$ inbalgt@clalit.org.il )

Ben-Gurion University of the Negev

Neta Loewenthal

Soroka Medical Center

Asher Tal

Ben-Gurion University of the Negev

Yotam Dizitzer

Soroka Medical Center

Yael Baumfeld

Ben-Gurion University of the Negev

Aviv Goldbart

Ben-Gurion University of the Negev

\section{Research Article}

Keywords: bronchiolitis, vitamin D deficiency, vitamin D status, children

Posted Date: January 7th, 2021

DOI: https://doi.org/10.21203/rs.3.rs-139230/v1

License: (1) This work is licensed under a Creative Commons Attribution 4.0 International License.

Read Full License 


\section{Abstract}

Objective: To evaluate serum $25(\mathrm{OH})$ vitamin $\mathrm{D}$ levels in infants and toddlers with acute bronchiolitis $(A B)$, compared to subjects with non-respiratory febrile illness.

Methods: a prospective cross-sectional case-control study which compared serum $25(\mathrm{OH})$ vitamin D levels between infants and toddlers diagnosed with Acute Bronchiolitis (AB) to subjects with nonrespiratory febrile illness.

Results: 127 patients aged $<24$ months were recruited; 80 diagnosed with $A B$ and 47 patients with nonrespiratory febrile illnesses. Both groups had similar demographics aside from age (mean of 7 months vs. 10 months in the $A B$ group compared to control group $(p=0.03)$ ) and respiratory symptoms. Serum $25(\mathrm{OH})$ vitamin D levels were significantly lower in the bronchiolitis group; median [IQR] 28[18-52] vs. $50[25-79] \mathrm{nmol} / \mathrm{L}$, respectively, $(p=0.005)$. Deficient vitamin $D$ levels $(<50 \mathrm{nmol} / \mathrm{L})$ was found more frequently in $A B$ than controls; $73 \%$ vs. $51 \%(p=0.028)$. Multivariate logistic regression, adjusted for age, sex, ethnicity and nutrition, showed vitamin D deficiency was more probable in $A B$ patients; OR[95\% $\mathrm{Cl}]$ 3.139[1.369-7.195]. No correlation was found between serum vitamin D levels and bronchiolitis severity, which was assessed via Modified Tal Score and by length of hospital stay.

Conclusion: children with acute bronchiolitis displayed significantly lower vitamin D levels than children with non-respiratory acute febrile illnesses.

\section{Background}

Acute bronchiolitis $(A B)$ is a major cause of morbidity during infancy and early childhood. The leading pathogen of acute bronchiolitis is respiratory syncytial virus (RSV) ${ }^{1}$. The importance of vitamin $D$ is well described; it plays a key role in the activation of the innate immune system ${ }^{2}$, particularly during lower respiratory tract infections ${ }^{3-5}$. Vitamin $D$ increases mucociliary clearance, regulate epithelial cell production and modulate inflammatory pathways ${ }^{6}$.

.Previous studies have suggested that cord $25(\mathrm{OH})$ vitamin D levels are significantly and inversely associated to the prevalence of respiratory infections and childhood wheezing ${ }^{7}$.Furthermore, vitamin $\mathrm{D}$ deficiency was associated with acute bronchiolitis ${ }^{8,9}$, poor asthma control ${ }^{10}$, severe asthma exacerbations ${ }^{11}$, and high consumption of asthma medications. To understand the role of vitamin $D$ in acute bronchiolitis further, we examined serum levels of $25(\mathrm{OH})$ vitamin $\mathrm{D}$ in infants younger than 24 months, that were clinically diagnosed with $A B$. We compared those patients to a control group of patients that had non-respiratory febrile illnesses such as urinary tract infection, acute gastroenteritis and dysentery. This study evaluated vitamin D status during acute respiratory illness in comparison to nonrespiratory acute febrile illness. We hypothesized that children with acute respiratory illness, such as acute bronchiolitis, will have lower vitamin D levels, since vitamin D and its receptor (VDR) were found in the epithelial cells of respiratory tract and influence mucocilliary clearness. We also investigated whether 
25(OH) vitamin D levels were correlated with bronchiolitis severity, measured with the Modified Tal Score (MTS) ${ }^{12}$ or with the length of hospital stay (LOS).

\section{Methods}

\section{Setting, subjects and data}

This prospective cross-sectional case-control study was conducted at Soroka University Medical Center (SUMC), the single tertiary medical center in Southern Israel, with a catchment population of approximately 750,000 . The Division of Pediatrics in SUMC contains 100 hospital beds with an annual activity of 35,000 ER visits and 12,000 hospitalizations; within them, approximately 400 admissions due to $A B$.

Infants and toddlers 24 months old and younger, who were referred to the ER at SUMC with febrile illness, during winter season, were invited to participate in our study. Children whose legal caregivers signed an informed consent form and who met none of the exclusion criteria were recruited. Exclusion criteria were: children suffering from chronic lung disease, congenital heart disease, inborn errors of metabolism, psychomotor retardation, hypotonia or any muscular abnormalities.

Upon clinical evaluation and initial treatment in the ER, children who were diagnosed with $A B$ were defined as cases, whereas children with febrile illnesses, but without respiratory symptoms, such as urinary tract infection or acute gastroenteritis, were defined as controls.

Bronchiolitis patients underwent bronchiolitis severity assessment in the ER setting using the Modified Tal Score (MTS). Bronchiolitis severity was defined by the following: scores $\leq 5$, mild; scores 6 to 10 , moderate; and scores $\geq 11$, severe. Bronchiolitis patients also underwent a nasal wash to assess respiratory viral antigens.

Questionnaires regarding demographical data, past medical history, Vitamin D administration, risk factors for asthma and Vitamin D deficiency and family history, were filled for all patients upon referral to the ER. Blood samples for Vitamin D serum levels were obtained.

Participants were followed throughout their clinical course of treatment and clinical data was recorded and documented. Collected data included whether patients were discharged from the ER or admitted to the hospital, length of stay upon hospitalization, admission to the pediatric intensive care unit (PICU) and need for mechanical ventilation.

All methods in the study were carried out in accordance with the Helsinki guidelines and declaration or any other relevant guidelines. The study was approved and overseen by the Institutional Review Board Committee of SUMC (Number- 5122). Study outcomes 
The primary outcome was the level of serum $25(\mathrm{OH})$ Vitamin D compared between bronchiolitis and nonbronchiolitis patients. Secondary outcome was the association between disease severity and serum Vitamin D levels within bronchiolitis. Disease severity was assessed by length of stay upon admission to the hospital for all patients and bronchiolitis severity assessment via MTS for bronchiolitis patients only.

\section{Laboratory measurements}

Blood samples were drawn from all subjects upon admission. Samples were refrigerated at $4{ }^{\circ} \mathrm{C}$ and then centrifuged within $24 \mathrm{~h}$. The serum was stored at $-80^{\circ} \mathrm{C}$ until analysis. Levels of $25(\mathrm{OH})$ vitamin D were measured in duplicate, with the Diasorin chemiluminescence immunoassay. We categorized $25(\mathrm{OH})$ vitamin D levels as: deficient: $<50 \mathrm{nmol} / \mathrm{L}$ : insufficient: $\geq 50$ and $<75 \mathrm{nmol} / \mathrm{L}$; and sufficient: $\geq 75 \mathrm{nmol} / \mathrm{L}$. We performed a nasal wash for participants of the bronchiolitis group to recover respiratory viral antigens, which were assessed with PCR.

\section{Statistical analysis}

We described and compared demographical and clinical characteristics and serum vitamin $25(\mathrm{OH}) \mathrm{D}$ levels between cases and controls. Variables were described as following - continuous variables with normal distribution as mean $\pm S D$, continuous variables with non-normal distribution or ordinal variables as median and interquartile range (IQR) and categorical data as sum and percentage. Univariate comparison between groups was performed with appropriate tests. Specifically, nominal variables were compared via Pearson's chi-square test; continuous variables that fulfilled parametric criteria were compared via Student's t-test; and ordinal variables and continuous non-parametric variables were compared via Wilcoxon or Mann-Whitney U tests.

Furthermore, we designed a multivariate logistic regression model to assess the risk of vitamin $D$ deficiency in the bronchiolitis patients, compared to controls, adjusted to age, sex, ethnicity and means of nutrition (whether they were breast fed, formula fed or both). Results are presented as Odds Ratio (OR) with $95 \%$ confidence interval $(\mathrm{Cl})$ and depicted via forest plot graph.

Third, we evaluated correlations between serum $25(\mathrm{OH})$ vitamin D levels and LOS upon admission, for all hospitalized patients. For bronchiolitis patients only we evaluated correlations between serum $25(\mathrm{OH})$ vitamin D levels and bronchiolitis severity (assessed by MTS severity score). Correlation was calculated via Pearson's chi square for parametric variables and via Spearman's correlation coefficient for nonparametric variables.

For all analyses, two-tailed p-values $\leq .05$ were considered statistically significant. Data were analyzed with IBM SPSS@ statistics software package version 23 .

\section{Results}

\section{Patient characteristics}


A total of 127 participants were recruited, including $80(63 \%)$ in the bronchiolitis group and $47(37 \%)$ in the control group (febrile, non-bronchiolitis). The groups had similar demographic details (Table 1) aside from age. Bronchiolitis patients were shown to be younger than febrile non-bronchiolitis patients; mean ( \pm SD) $7 \pm 6$ months and $10 \pm 6$ months, respectively, ( $p$ 0.003). The groups had similar percentages of allergic rhinitis, atopic dermatitis, and cow's milk protein allergy. More than half of the subjects in the bronchiolitis (59\%) and control (51\%) groups were of Bedouin origin. According to the guidelines of the Israeli Health Ministry, children under the age of one year are recommended to be treated with supplementary vitamin D, $400 \mathrm{IU}$ per day. However, only $66 \%$ of the bronchiolitis group and $60 \%$ of the control group received vitamin D. regarding to previous medical history, nearly one third of patients (33\% and $30 \%$, respectively) were treated in the past with beta agonist inhalations. $23 \%$ of children in both groups had previously received systemic steroids.

Regarding clinical characteristics of admitted patients, bronchiolitis patients had a significantly longer LOS than non-bronchiolitis; median [IQR] 4 [2-6] days and 1 [0-3] days, respectively, $p<0.001$.

\section{Serum Vitamin D levels}

Serum 25(OH) vitamin D levels were lower in the bronchiolitis group; median [IQR] 28 [18-52] nmol/L and 50 [25-79] nmol/L, respectively, p 0.005. Deficient $25(\mathrm{OH})$ vitamin $D$ levels $(<50 \mathrm{nmol} / \mathrm{L})$ were found more frequently in the bronchiolitis group than in the control group; $n$ (\%) 58/80 (73\%) and $24 / 57$ (51\%), respectively and sufficient $25(\mathrm{OH})$ vitamin $\mathrm{D}$ levels were found more frequently in the control group than in the bronchiolitis group; $n$ (\%) 13/47 (28\%) and 9/80 (11\%), respectively, p 0.028 (Table 2).

Nasal washes for viral antigen were performed in 72 of 80 bronchiolitis diagnosed subjects $(90 \%)$. Among these 72 subjects, we detected RSV in $71 \%(n=51)$, human metapneumo virus in $11 \%(n=8)$, influenza A virus in $8 \%(n=6)$, adenovirus in $3 \%(n=2)$, one RSV \& corona virus co-infection $(n=1)$, one RSV\& influenza A virus co-infection $(n=1)$, and negative results from a respiratory viral panel in $7 \%(n=$ 5).

\section{Bronchiolitis and Vitamin D deficiency}

Multivariate logistic regression, adjusted for age, sex, ethnicity and nutrition, showed that bronchiolitis patients were more likely to have Vitamin $D$ deficiency compared to febrile non-bronchiolitis patients; OR [95\%Cl] 3.139 [1.369-7.195] (Fig. 1).

For the bronchiolitis group, no statistically significant correlation was found between serum vitamin $D$ levels and bronchiolitis severity via MTS; Spearman's rho $-0.189, \mathrm{p} 0.095$. However, bronchiolitis patients showed higher percentages of children with vitamin $D$ deficiency divided by levels of clinical severity depicted by MTS (Fig. 2). For bronchiolitis and non-bronchiolitis patients, no statistically significant correlation was found between serum Vitamin D levels and LOS; Spearman's rho - 0.143, p 0.204 and 0.052 , p 0.726, respectively. However, bronchiolitis and non-bronchiolitis patients both showed higher percentages of serum Vitamin D deficiency within patients with longer LOS (Fig. 3a and 3b, respectively). 


\section{Disscussion}

Our results demonstrated that vitamin D deficiency was associated with acute bronchiolitis, compared to febrile non-bronchiolitis illnesses, when adjusted to age, sex, ethnicity and means of nutrition. Previous studies have reported inconsistent findings regarding the role of vitamin D status in acute bronchiolitis.

A potential explanation for why a vitamin $D$ deficiency was associated with an acute respiratory tract infection might be related to its important role in the innate immune system. Vitamin $D$ is involved in inducing the activity of the endogenic antimicrobial proteins, defensins and cathelicidin, which fight against bacterial and viral infections ${ }^{13,14}$ in bronchial epithelial cells ${ }^{15}$. Wang et al ${ }^{16}$ showed that vitamin $D\left(1,25(\mathrm{OH})_{2} D_{3}\right)$ could directly induce the cathelicidin antimicrobial peptide and defensin $\beta_{2}$, which suggested that it could provide enhanced antimicrobial protection against infections. This protection accelerated healing after surgery. Janssen et al ${ }^{17}$ demonstrated a genetic association between polymorphisms in the vitamin D receptor and the severity of RSV bronchiolitis ${ }^{18}$. Other experimental studies have indicated that both ultraviolet B-ray exposure and oral vitamin D supplementation could raise the level of cathelicidin in skin ${ }^{19}$. It is known that vitamin $D$ status is determined largely from ultraviolet B-ray exposure; therefore, $25(\mathrm{OH})$ vitamin D levels are lower during winter, the peak season for acute bronchiolitis. Both groups were recruited during winter, thus eliminating season as a factor that influence vitamin D levels. Taken together, these clinical and mechanistic data supported our hypothesis that vitamin D might be an important modulator of the immune response to respiratory viruses in acute bronchiolitis. Moreover, our control group had acute infections, such as acute gastroenteritis or urinary tract infections, without respiratory symptoms. Thus, our findings suggested that vitamin D status played a specific protective role in respiratory tract infections, but not necessarily in other infections. On the other hand, Thornton et al reported that vitamin D deficiency was associated with increased rates of diarrhea with vomiting and with earache and/or discharge with fever, but was not significantly related to cough with fever in school-age children ${ }^{20}$.Different findings were brought up by Hassam et al ${ }^{21}$.that did not find association between vitamin $\mathrm{D}$ levels and diarrhea in children under five years of age.

We could not demonstrate a correlation between $25(\mathrm{OH})$ vitamin D levels and bronchiolitis severity (estimated with the MTS severity score) or LOS. Similar results were reported by Beigelman et $\mathrm{al}^{22}$, who studied 145 infants and concluded that the vitamin D status during acute bronchiolitis was not associated with indicators of bronchiolitis severity. Roth et $\mathrm{al}^{23}$ studied 120 hospitalized Canadian children under 2 years old, and found no association between vitamin D status and acute lower respiratory infections.

A potential explanation for this association might be that vitamin D can shift immunomodulatory effects towards T helper cell (Th)-2 responses ${ }^{24}$. In contrast, RSV bronchiolitis severity is inversely proportional to the Th-1 response ${ }^{25}$. This was observed when RSV was the causing pathogen. On the other hand, in a previous study on 28 Japanese children, more children with lower respiratory tract infection that required supplementary oxygen and ventilator management were also $25(\mathrm{OH})$ vitamin D deficient ${ }^{26}$. McNally 
reported that the mean vitamin $\mathrm{D}$ level was significantly lower in subjects with acute lower respiratory tract infections admitted to the pediatric intensive care unit, compared to children admitted to the general pediatrics ward ${ }^{27}$. That finding implied that vitamin $\mathrm{D}$ might be associated with severe respiratory illnesses. Indeed, among our 80 subjects with bronchiolitis, those admitted to the pediatric intensive care unit and those with prolonged hospitalizations had the lowest 25(OH) vitamin D levels. Moreover, we would like to emphasize that the $25(\mathrm{OH})$ vitamin D levels were taken upon admission; thus, the level was not influenced by the LOS.

Although we could not demonstrate a correlation between bronchiolitis severity and $25(\mathrm{OH})$ vitamin $\mathrm{D}$ deficiency, we found that two subjects with severe bronchiolitis (i.e., those with the MTS $\geq 11$ ) or subjects with the longest hospitalizations had insufficient levels of $25(\mathrm{OH})$ vitamin $D$. Therefore, vitamin $D$ could serve as a marker of severity. The correlation between $25(\mathrm{OH})$ vitamin D deficiency and bronchiolitis severity was demonstrated in a large multicenter cohort of 1016 infants that were hospitalized for acute bronchiolitis, with increased risk for hospitalization in intensive care units and for prolonged LOS $^{28}$.

Our main study limitation was the relatively small sample size. Nevertheless, we found a significant difference in $25(\mathrm{OH})$ vitamin $D$ levels between groups. Additionally, this study had a single-center design, with a specific population; more than half of participants were of Bedouin origin. This population, typically belong to a low socioeconomic status, with large families and overcrowding. They live in poor accommodations/housing conditions, and some Bedouin settlements have limited access to health care. However, we must stress that the $25(\mathrm{OH})$ vitamin D levels were not significantly different between Bedouin and non-Bedouin subjects; thus, ethnicity was not a bias in this study (Fig. 1).

Additional studies are needed to address the diagnostic and therapeutic implications of the role of vitamin $\mathrm{D}$ in respiratory morbidity, particularly in acute bronchiolitis. Future studies should be conducted in multiple centers to include a large number of patients that might better represent the general population.

Our findings suggested that an intervention that could change the vitamin D status in infants might be beneficial. A clear answer to this question could have important implications on public health in the future. Indeed, the prevalence of inadequate vitamin D status is generally high worldwide, particularly among at-risk subpopulation groups. Moreover, in young children, acute bronchiolitis is accompanied by substantial disease burden.

\section{Conclusion}

We found an association between $25(\mathrm{OH})$ vitamin D deficiency and acute bronchiolitis. This finding suggested that inadequate vitamin D status might serve as a risk factor for acute bronchiolitis. However, findings from this relatively small prospective study warrant further investigation on a larger scale and in different at-risk populations. Those studies might provide a more precise estimate of the potential impact of vitamin $\mathrm{D}$ status on acute respiratory infections in children. 


\section{Declarations}

Acknowledgement:

Not applicable

\section{Author Contributions:}

IGT: Contributed to conception and design, screened and enrolled patients, critically revised the manuscript for important and intellectual content, and gave final approval.

NL: Contributed to conception and design, drafted the manuscript.

AT: Contributed to conception and design, drafted the manuscript, and gave final approval.

YD: Contributed to acquisition, analysis and interpretation, critically revised the manuscript for important and intellectual content.

YB: Contributed to analysis, drafted the manuscript.

AG: Contributed to conception and design, critically revised the manuscript for important and intellectual content, and gave final approval.

Funding: no funding was received for conducting this study.

Conflicts of interest/Competing interests: the authors have no conflicts of interest to declare that are relevant to the content of this article.

Ethical approval: the study was approved by the institutional ethics committee (Soroka University Medical Center, Number- 5122).

Consent to participate: written informed consent was obtained from the parents of all participants included in the study.

Consent for publication: N/A

Availability of data and material: the authors confirm that the data supporting the findings of this study are available within the article and its supplementary materials.

Code availability: N/A

\section{References}

1. Collins CL, Pollard AJ. Respiratory syncytial virus infections in children and adults. J Infect 2002;45:10-17. 
2. Chesney RW. Vitamin D and The Magic Mountain: the anti-infectious role of the vitamin. J Pediatr 2010;156(5):698-703. doi:10.1016/j.jpeds.2010.02.002

3. Wayse V, Yousafzai A, Mogale K, Filteau S. Association of subclinical vitamin D deficiency with severe acute lower respiratory infection in Indian children under 5 y. Eur J Clin Nutr 2004;58:563-7.

4. Ginde AA, Mansbach JM, Camargo CA Jr. Association between serum 25-hydroxyvitamin D level and upper respiratory tract infection in the Third National Health and Nutrition Examination Survey. Arch Intern Med 2009;169:384-90.

5. Roth $\mathrm{DE}$, Jones $\mathrm{AB}$, Prosser $\mathrm{C}$, Robinson JL, Vohra S. Vitamin D receptor polymorphisms and the risk of acute lower respiratory tract infection in early childhood. $J$ Infect Dis 2008;197:676-680.

6. Akcan FA, Dündar Y, Akcan HB, Uluat A, Cebeci D, Ünlü I. Evaluation of nasal mucociliary clearance time in patients with vitamin-D deficiency. Eur Arch Otorhinolaryngol 2019;276:1075-80.

7. Camargo CA, Ingham T, Wickens K, Thadhani R, Silvers KM, Epton MJ, Town GI, Pattemore PK, Espinola JA, Crane J. Cord-Blood 25-Hydroxyvitamin D levels and Risk for Respiratory Infection, Wheezing, and Asthma. Pediatrics 2010;180-187DOI: 10.1542/peds.2010-0442.

8. Mansbach JM, Camargo CA Jr. Bronchiolitis: lingering questions about its definition and the potential role of vitamin D. Pediatrics 2008;122(1):177-9. doi: 10.1542/peds.2007-3323.

9. Belderbos ME, Houben ML, Wilbrink B, Lentjes E, Bloemen EM, Kimpen JL, Rovers M, Bont L. Cord blood vitamin $D$ deficiency is associated with respiratory syncytial virus bronchiolitis. Pediatrics 2011;127(6):e1513-20. doi: 10.1542/peds.2010-3054.

10. Chinellato I, Piazza M, Sandri M, Peroni D, Piacentini G, Boner AL. Vitamin D serum levels and markers of asthma control in Italian children. J Pediatr. 2011;158(3):437-441. doi:10.1016/j.jpeds.2010.08.043

11. Brehm JM, Schuemann B, Fuhlbrigge AL, Hollis BW, Sf Robert, Zeiger S. Weiss ST, Litonjua AA, for the Childhood Asthma Management Program Research Group. Serum vitamin D levels and severe asthma exacerbations in the Childhood Asthma Management Program study. J Allergy Clin Immunol 2010;126:52-8.

12. Golan-Tripto I, Goldbart A, Akel K, Dizitzer Y, Novack V, Tal A. Modified Tal Score: Validated score for prediction of bronchiolitis severity. Pediatr Pulmonol 2018; 53: 796- 801. https://doi.org/10.1002/ppul.24007

13. Ganz T. Defensins: antimicrobial peptides of innate immunity. Nat Rev Immunol 2003;3(9):710-720.

14. Leikina E, Delanoe-Ayari $\mathrm{H}$, Melikov $\mathrm{K}$, et al. Carbohydrate binding molecules inhibit viral fusion and entry by crosslinking membrane glycoproteins. Nat Immunol 2005;6(10):995-1001.

15. Yim S, Dhawan P, Ragunath C, Christakos S, Diamond G. Induction of cathelicidin in normal and CF bronchial epithelial cells by 1,25- dihydroxyvitamin D(3). J Cyst Fibros 2007; 6(6):403- 410.

16. Wang TT, Nestel FP, Bourdeau V, et al. Cutting edge: 1,25-dihydroxyvitamin D3 is a direct inducer of antimicrobial peptide gene expression. J Immunol 2004;173(5):2909-12. 
17. Janssen R, Bont L, Siezen CL, et al. Genetic susceptibility to respiratory syncytial virus bronchiolitis is predominantly associated with innate immune genes. J Infect Dis. 2007;196(6): 826-834

18. Roth $D E$, Jones $A B$, Prosser $C$, et al. Vitamin $D$ receptor polymorphisms and the risk of acute lower respiratory tract infection in early childhood. $J$ Infect Dis 2008;197:676-80.

19. Glaser R, Navid F, Schuller W, et al. UV-B radiation induces the expression of antimicrobial peptides in human keratinocytes in vitro and in vivo. J Allergy Clin Immunol 2009;123(5):1117-1123.

20. Thornton KA, Marín C, Mora-Plazas M, Villamor E. Vitamin D deficiency associated with increased incidence of gastrointestinal and ear infections in school-age children. Pediatr Infect Dis J. 2013 Jun;32(6):585-93. doi: 10.1097/INF.0b013e3182868989. PMID: 23340562.

21. Hassam I, Kisenge R, Aboud S, Manji K. Association of vitamin D and diarrhoea in children aged less than five years at Muhimbili national hospital, Dar es Salaam: an unmatched case control study. BMC Pediatr. 2019 Jul 15;19(1):237. doi: 10.1186/s12887-019-1614-4. PMID: 31307425; PMCID: PMC6628469.

22. Beigelman A, Castro M, Schweiger TL, et al. Vitamin D Levels Are Unrelated to the Severity of Respiratory Syncytial Virus Bronchiolitis Among Hospitalized Infants. J Pediatric Infect Dis Soc 2015;4(3):182-188. doi:10.1093/jpids/piu042

23. Roth DE, Jones AB, Prosser C, Robinson JL, Vohra S. Vitamin D status is not associated with the risk of hospitalization for acute bronchiolitis in early Childhood. Eur J Clin Nutr 2009; 63:297-299. doi:10.1038/sj.ejcn.1602946

24. Rausch-Fan X, Leutmezer F, Willheim M, et al. Regulation of cytokine production in human peripheral blood mononuclear cells and allergen-specific Th cell clones by 1-alpha-25- dihydroxyvitamin D3. Int Arch Allergy Immunol 2002; 128, 33-41.

25. Bont L, Heijnen CJ, Kavelaars A, et al. Local interferon-gamma levels during respiratory syncytial virus lower respiratory tract infection are associated with disease severity. J Infect Dis 2001; 184, 355-358.

26. Inamo $Y$, Hasegawa $M$, Saito $K$, et al. Serum vitamin D concentrations and associated severity of acute lower respiratory tract infections in Japanese hospitalized children. Pediatr Int 2011;53(2):199201.

27. McNally JD, Leis K, Matheson LA, Karuananyake C, Sankaran K, Rosenberg AM. Vitamin D Deficiency in Young Children With Severe Acute Lower Respiratory Infection. Pediatr Pulmonol 2009;44:981-988.

28. Vo Ph, Koppel C, Espinola J, Mansbach J, Celedón J, Hasegawa K, Bair-Merritt M, Camargo C. Vitamin D Status at the Time of Hospitalization for Bronchiolitis and Its Association with Disease Severity. J Pediatr 2018;203:416-422.e1. doi: 10.1016/j.jpeds.2018.07.097

\section{Tables}

Table 1 - Demographic and clinical characteristics of infants in the bronchiolitis and control groups 


\begin{tabular}{|c|c|c|c|}
\hline \multirow[t]{2}{*}{ Characteristic } & Bronchiolitis Group & Control Group & \multirow[t]{2}{*}{$\mathrm{p}$ value } \\
\hline & $\mathrm{N}=80$ & $\mathrm{~N}=47$ & \\
\hline \multicolumn{4}{|l|}{ Demographic charachteristics } \\
\hline Age, months ${ }^{a}$ & $7 \pm 6$ (months) & $10 \pm 6$ (months) & 0.003 \\
\hline Sex (male) ${ }^{b}$ & $47(58 \%)$ & $23(49 \%)$ & 0.283 \\
\hline Ethnicity (Bedouin) ${ }^{b}$ & $47(59 \%)$ & $24(51 \%)$ & 0.400 \\
\hline Attend day care ${ }^{b}$ & $21(28 \%)$ & $16(34 \%)$ & 0.451 \\
\hline \multicolumn{4}{|l|}{ Medical history and clinical characteristics } \\
\hline Prematurity ( $\leq 37$ weeks gestation) ${ }^{b}$ & $9(11 \%)$ & $5(11 \%)$ & 0.915 \\
\hline \multicolumn{4}{|l|}{ Nutrition ${ }^{\mathrm{b}}$ : } \\
\hline Exclusive breast feeding (until 4 mo.) & $22(28 \%)$ & $13(25 \%)$ & \multirow[t]{3}{*}{0.619} \\
\hline Formula & $18(23 \%)$ & $16(31 \%)$ & \\
\hline Breast feeding and formula & $38(49 \%)$ & $23(44 \%)$ & \\
\hline Vitamin $\mathrm{D}$ administration ${ }^{\mathrm{b}}$ & $53(66 \%)$ & $28(60 \%)$ & 0.450 \\
\hline Passive exposure to smoking ${ }^{b}$ & $27(37 \%)$ & $17(36 \%)$ & 0.981 \\
\hline $1^{\text {st }}$ degree relative with asthma ${ }^{b}$ & $36(47 \%)$ & $11(23 \%)$ & 0.008 \\
\hline Allergic rhinitis ${ }^{b}$ & $5(6 \%)$ & $1(2 \%)$ & 0.290 \\
\hline Atopic dermatitis ${ }^{b}$ & $3(4 \%)$ & $2(4 \%)$ & 0.888 \\
\hline Cow's milk protein allergy ${ }^{b}$ & $2(3 \%)$ & $4(9 \%)$ & 0.123 \\
\hline Previous respiratory episode ${ }^{b}$ & $23(29 \%)$ & $15(32 \%)$ & 0.707 \\
\hline Previous systemic steroid treatment ${ }^{b}$ & $18(23 \%)$ & $11(23 \%)$ & 0.907 \\
\hline Previous treatment with beta agonists ${ }^{b}$ & $26(33 \%)$ & $15(32 \%)$ & 0.946 \\
\hline \multicolumn{4}{|l|}{ Hospitalization associated characteristics } \\
\hline Length of stay (days) ${ }^{c}$ & $4[2-6]$ & $1[0-3]$ & $<0.001$ \\
\hline
\end{tabular}

Note. Table 1 shows population characteristics including demographic characteristics, medical history and clinical characteristics. Univariate analysis was held to assess the difference between bronchiolitis vs. control groups. ${ }^{\text {a. }}$ variables are depicted by Mean $\pm S D$ and compared via Students' T-test; $^{\text {b. }}$ : variables 
are depicted by $\mathrm{n}(\%)$ and compared via Pearson's Chi-square; ${ }^{\mathrm{c}}$ : variables are depicted by Median and [IQR] and compared via Man-Whitney U test. Statistical significance is represented by $p$ value.

Table 2 - Serum 25(OH) vitamin D levels levels in infants in the bronchiolitis and control groups

\begin{tabular}{|c|c|c|c|}
\hline & $\begin{array}{l}\text { Bronchiolitis Group } \\
\mathrm{N}=80\end{array}$ & $\begin{array}{l}\text { Control Group } \\
\mathrm{N}=47\end{array}$ & $p$ value \\
\hline Serum Vitamin D levels $(\mathrm{nmol} / \mathrm{L})^{c}$ & 28 [18-52] & 50 [25-79] & 0.005 \\
\hline Vitamin $\mathrm{D}$ catagories ${ }^{\mathrm{b}}$ : & & & 0.028 \\
\hline Deficient (Vitamin D: $\leq 50 \mathrm{nmol} / \mathrm{L})$ & $58(73 \%)$ & $24(51 \%)$ & \\
\hline Insufficient (Vitamin D: $50-75 \mathrm{nmol} / \mathrm{L}$ ) & $13(16 \%)$ & $10(21 \%)$ & \\
\hline Sufficient (Vitamin D: $\geq 75 \mathrm{nmol} / \mathrm{L}$ ) & $9(11 \%)$ & $13(28 \%)$ & \\
\hline
\end{tabular}

Note. Table 2 shows levels of serum vitamin D and vitamin D categories. Univariate analysis was held to assess the difference between bronchiolitis vs. control groups. ${ }^{a}$ : variables are depicted by Mean $\pm S D$ and compared via Students' T-test; ${ }^{\text {b: }}$ variables are depicted by $\mathrm{n}(\%)$ and compared via Pearson's Chi-square; : variables are depicted by Median and [IQR] and compared via Man-Whitney $U$ test. Statistical significance is represented by $p$ value.

\section{Figures}




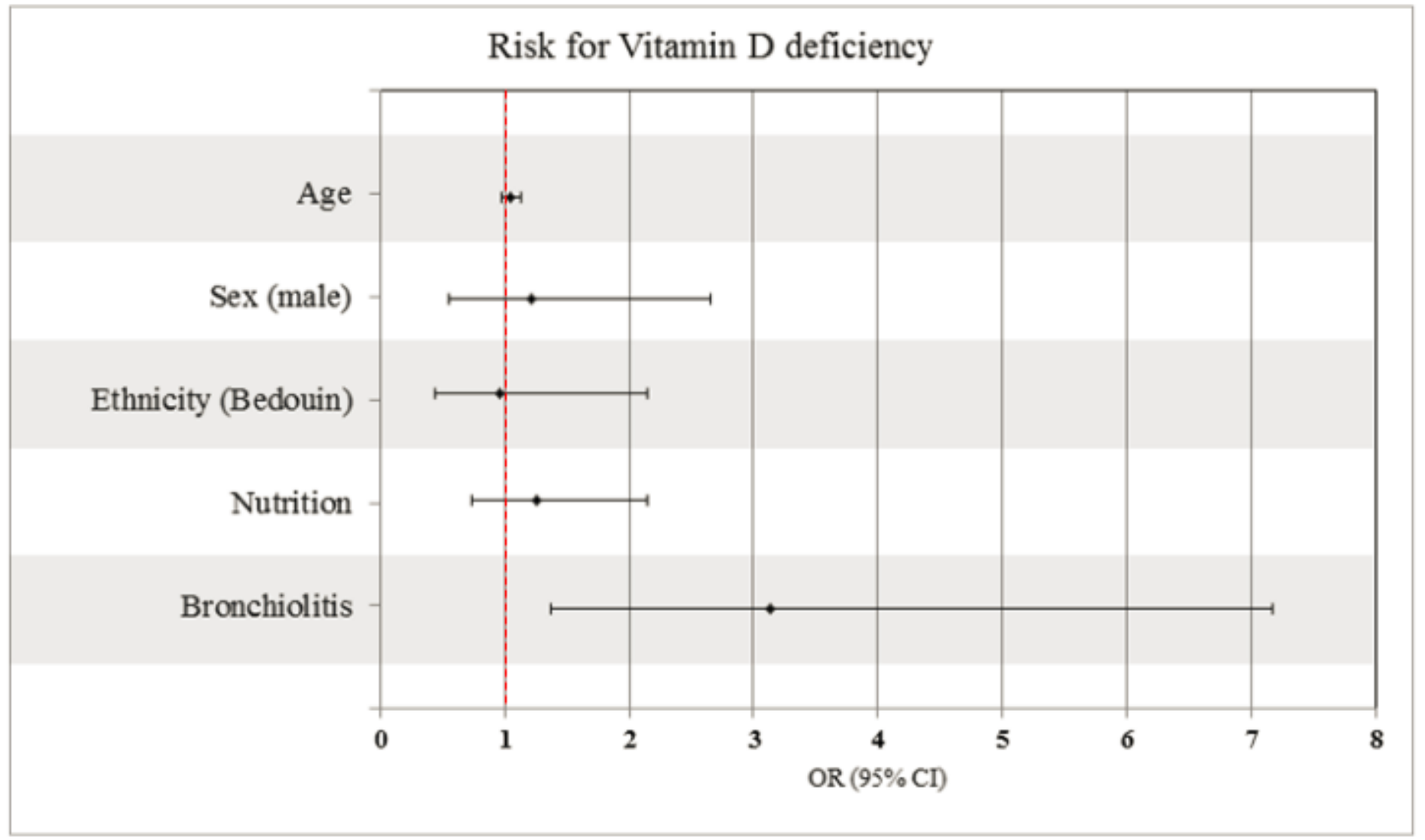

Figure 1

Forest plot for multivariate logistic regression assessing Odds Ratio for Vitamin D deficiency among the bronchiolitis and control group adjusted for age, sex, ethnicity and means of nutrition. 


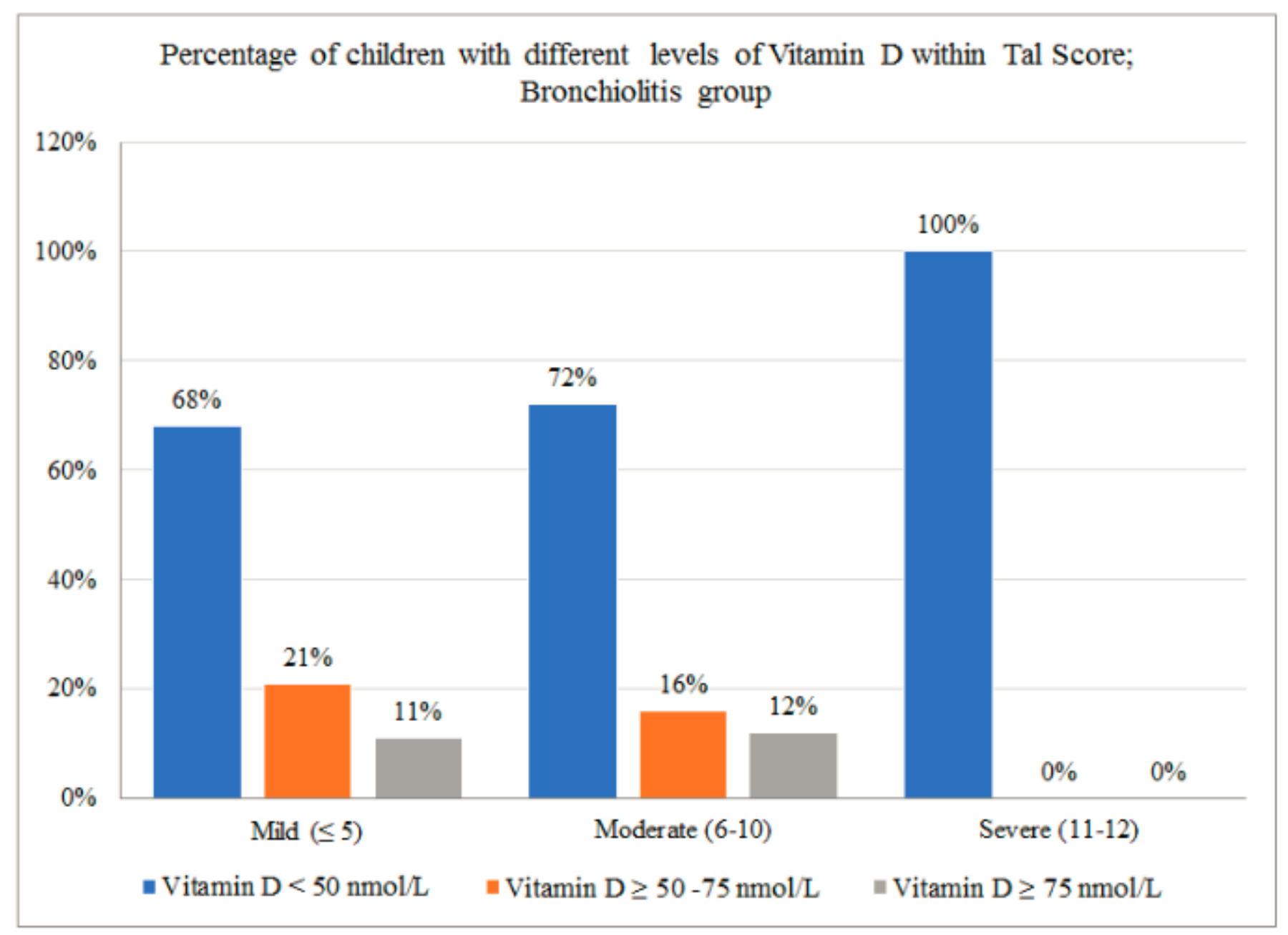

Figure 2

Different 25(OH) vitamin D levels within grouped modified Tal score in the bronchiolitis group $(n=79)$.
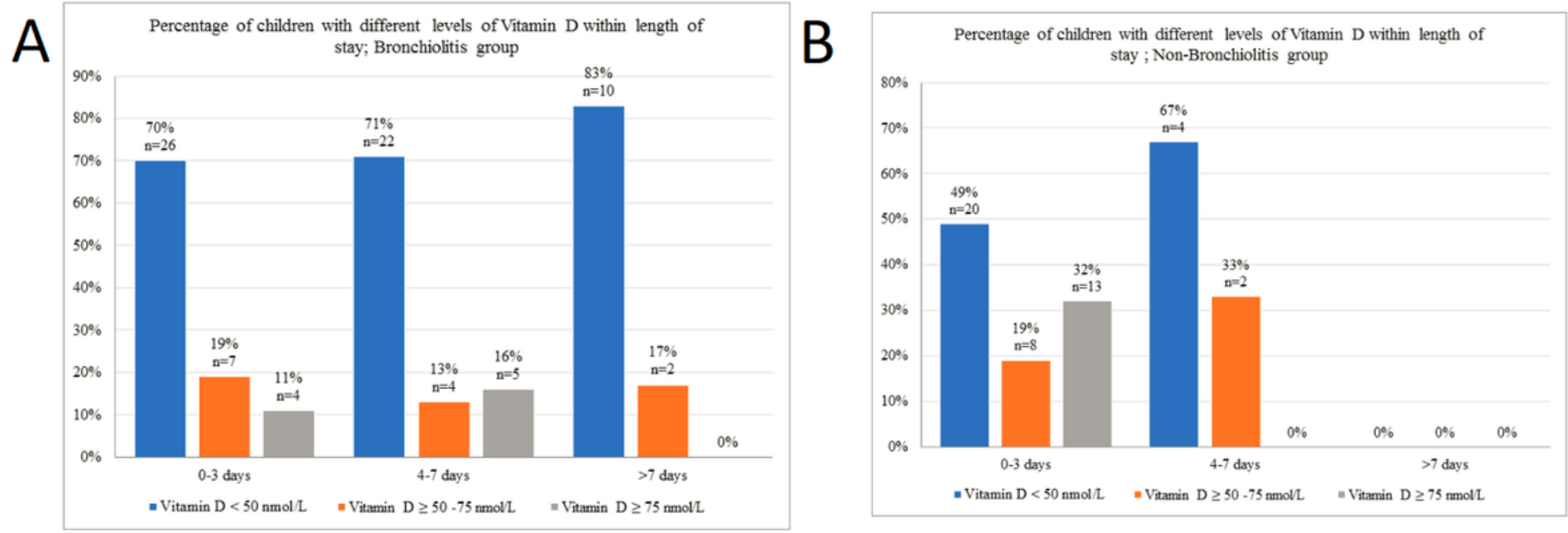

Figure 3 
Different 25(OH) vitamin D levels, grouped by length of stay in bronchiolitis group $(n=79)$. Different $25(\mathrm{OH})$ vitamin $\mathrm{D}$ levels, grouped by length of stay in non-bronchiolitis group $(n=47)$. 